\title{
Synchrony and idiosyncrasy in the gut microbiome of wild primates
}

Authors: Johannes R. Björk ${ }^{1 *}$, Mauna R. Dasari ${ }^{1}$, Kim Roche ${ }^{2}$, Laura Grieneisen ${ }^{3}$, Trevor J. Gould $^{3}$, Jean-Christophe Grenier ${ }^{4,5}$, Vania Yotova ${ }^{4}$, Neil Gottel ${ }^{6}$, David Jansen ${ }^{1}$, Laurence R. Gesquiere $^{7}$, Jacob B. Gordon ${ }^{7}$, Niki H. Learn ${ }^{8}$, Tim L. Wango ${ }^{9,10}$, Raphael S. Mututua ${ }^{9}$, J. Kinyua Warutere ${ }^{9}$, Long'ida Siodi ${ }^{9}$, Sayan Mukherjee ${ }^{2}$, Luis B. Barreiro ${ }^{11}$, Susan C.

Alberts $^{7,12,13}$, Jack A. Gilbert ${ }^{6}$, Jenny Tung ${ }^{7,12,13,14}$, Ran Blekhman ${ }^{3,15}$, Elizabeth A. Archie ${ }^{1 *}$

Affiliations:

$9{ }^{1}$ Department of Biological Sciences, University of Notre Dame, Notre Dame, IN 46556, USA

${ }^{2}$ Program in Computational Biology and Bioinformatics, Duke University, Durham, NC 27708, 11 USA

$12{ }^{3}$ Department of Genetics, Cell Biology, and Development, University of Minnesota,

13 Minneapolis, MN 55455, USA

$14{ }^{4}$ Department of Genetics, CHU Sainte Justine Research Center, Montréal, QC, H3T1C5,

15 Canada.

$16{ }^{5}$ Research Center, Montreal Heart Institute, Montréal, Quebec H1T 1C8, Canada

$17{ }^{6}$ Department of Pediatrics and the Scripps Institution of Oceanography, University of California,

18 San Diego, San Diego, CA 92093, USA

$19{ }^{7}$ Department of Biology, Duke University, Durham, NC 27708, USA

$20{ }^{8}$ Department of Ecology and Evolutionary Biology, Princeton University, Princeton, NJ 08544, 21 USA

$22{ }^{9}$ Amboseli Baboon Research Project, Amboseli National Park, Kenya

$23{ }^{10}$ The Department of Veterinary Anatomy and Animal Physiology, University of Nairobi, Kenya

$24{ }^{11}$ Department of Medicine, Section of Genetic Medicine, University of Chicago, Chicago, IL

25 60637, USA

$26{ }^{12}$ Department of Evolutionary Anthropology, Duke University, Durham, NC 27708, USA

$27{ }^{13}$ Duke University Population Research Institute, Duke University, Durham, NC 27708, USA

$28{ }^{14}$ Canadian Institute for Advanced Research, Toronto, Ontario M5G 1M1, Canada

$29{ }^{15}$ Department of Ecology, Evolution, and Behavior, University of Minnesota, Minneapolis, MN

30 55455, USA

$31 *$ Correspondence to: bjork.johannes@gmail.com; earchie@nd.edu 
32 Abstract: Human gut microbial dynamics are highly individualized, making it challenging to

33 link microbiota to health and to design universal microbiome therapies. This individuality is

34 typically attributed to variation in diets, environments, and medications, but it could also emerge

35 from fundamental ecological forces that shape primate microbiota more generally. Here we

36 leverage extensive gut microbiome time series from wild baboons-hosts who experience little

37 interindividual dietary and environmental heterogeneity — to test whether gut microbial dynamics

38 are synchronized across hosts or largely idiosyncratic. Despite their shared lifestyles, we find

39 strong evidence for idiosyncrasy. Over time, samples from the same baboon were much more

40 similar than samples from different baboons, and host-specific factors collectively explained

$4130 \%$ of the deviance in microbiome dynamics, compared to just $3 \%$ for factors shared across

42 hosts. Hence, individualization may be common to mammalian gut microbiota, and designing

43 universal microbiome interventions may face challenges beyond heterogeneity in human

44 lifestyles.

Introduction

Mammalian gut microbiomes are highly complex, dynamic ecosystems. From these dynamics emerge a set of life-sustaining services for hosts, which help them digest food, process toxins, and resist invading pathogens. Despite their importance, our understanding of gut microbial dynamics, especially the collective dynamics of microbial communities from hosts

51 living in the same population, is remarkably poor (1). This gap exists in part because we lack

52 time series data that track gut microbiota longitudinally across many hosts in the same

53 population. As a result, we cannot answer key questions. For example, when host populations

54 encounter shifting environments and resources, does each host's microbiota respond similarly-

55 i.e., in synchrony-or idiosyncratically to these changes? Further, are microbial dynamics

56 especially similar when hosts live in the same social unit or have shared traits, such as age, sex or $57 \quad$ social status?

Answering these questions is important because synchronized host microbiomes could 59 help explain shared microbiome-associated traits in host populations, such as patterns of disease 60 susceptibility $(2,3)$. A high degree of microbiome synchrony could also be good news for 61 researchers working to predict microbiome dynamics because it would suggest that similar 
62 ecological principles govern microbiome dynamics across hosts (4). There is also theoretical

63 justification to expect some degree of synchrony, as host populations and their microbiomes can

64 be considered a 'microbiome metacommunity' (see e.g., 5, 6-8). Metacommunity theory predicts

65 that synchrony will arise across microbiomes if hosts experience similar environmental

66 conditions and/or high rates of microbial dispersal between each host's microbiome $(9,10)$.

However, even in the presence of synchronizing forces like shared environments and

68 high rates of microbial dispersal, there are many reasons why hosts in a microbiome

69 metacommunity could exhibit idiosyncratic (i.e., individualized) microbiome compositions and

70 dynamics. Idiosyncratic dynamics are expected when the same microbes in different hosts

71 respond in different ways to environmental fluctuations, chance events, and/or interactions with

72 other microbes (11-14). These forces are likely to be important in the gut microbiome where

73 priority effects, functional redundancy, and horizontal gene flow can cause the same microbial

74 taxon to perform different functions, play different ecological roles, and exhibit different

75 environmental responses in different hosts $(15,16)$. Furthermore, in humans, gut microbiome

76 dynamics are often described as "personalized" $(17,18)$. However, personalized dynamics in

77 humans are nearly always attributed to large interpersonal differences in diet, medications, and

78 lifestyles (19-22), and not to fundamentally different microbiome responses to the environment

79 itself (19). If personalized dynamics persist in a different primate species, even in the presence of

80 shared environments, this pattern would suggest that: (i) host-specific dynamics are a common

81 feature of primate gut microbial communities (i.e., are not unique to humans and are not solely

82 attributable to large interpersonal differences in human lifestyles); (ii) predicting gut microbial

83 dynamics in individual hosts may prove difficult; and (iii) microbiome interventions to improve

84 human health may face challenges beyond heterogeneity in human lifestyles, and instead may be

85 related to the fundamental ecological principles that govern the gut microbiome.

\section{Data and methods}

Here we test the degree to which gut microbiome compositions and dynamics in a host

89 population are synchronized versus idiosyncratic using extensive time series data from a

90 population of wild baboons in the Amboseli ecosystem in Kenya (23). Baboons are terrestrial

91 primates that live in stable social groups, typically with 20 to 130 members. The 600 baboons in 
92 our data set lived in 12 social groups over a 14-year span (April 2000 to September 2013; 5

93 original groups and 7 groups that were fission/fusion products from these original groups; Fig.

94 1A). The baboons were members of the well-studied Amboseli baboon population (23), which

95 has been studied by the Amboseli Baboon Research Project since 1971. This project collected

96 detailed longitudinal data on the weather the animals experienced; their social group

97 memberships, ranging patterns and diets; and host traits such as age, sex, social relationships,

98 and dominance ranks (see Supplementary Materials).

99 Importantly, like many natural host populations, the Amboseli baboons experience shared

100 diets, environments, and opportunities for between-host microbial dispersal that could drive

101 microbiome synchrony across hosts. Because baboons are not territorial, all 12 baboon social

102 groups used an overlapping $\sim 60 \mathrm{~km}^{2}$ range (Fig. 1B; video S1; (24)). Hence all animals were

103 exposed to similar microbes from the environment and shared seasonal changes in rainfall and

104 temperature (24-26). The Amboseli ecosystem is a semi-arid savanna where very little rain falls

105 from June to October, with highly variable rainfall between November and May (Fig. 1C; mean

106 annual rainfall between 2000 and 2013 was $319 \mathrm{~mm}$; range $=140 \mathrm{~mm}$ to $559 \mathrm{~mm}$ ). These

107 seasonal shifts in climate drive a rotating set of foods consumed by the baboons: during the dry

108 season the baboons rely largely on grass corms, shifting to growing grass blades and grass seed

109 heads in the wet season (Fig. 1D). Within baboon social groups, diets and environments are

110 especially congruent because group members travel together in a coordinated fashion across the

111 landscape, encountering and consuming resources and feeding on the same seasonally available

112 foods at the same time $(24,27-31)$. Group members also groom each other, combing through

113 each other's fur and placing some items in their mouths, which may contribute to host-to-host

114 microbial transmission (32). Finally, at the level of individual hosts, host genetic variation has a

115 consistent, albeit modest, effect on gut microbiome composition in this population (24). Other

116 host-specific traits, like age, sex, and social status, also lead some individuals to share aspects of

117 their behavior, immune profiles, and physiology, which could also lead to more congruent

118 microbiome dynamics.

119 A key advance in our study is longitudinal sampling of gut microbial composition via

120 16S rRNA gene sequencing from fecal samples collected from hundreds of known baboons

121 throughout their lives (Fig. 1A). Such dense, long-term, longitudinal microbiome sampling is

122 difficult to achieve in many animals, including humans. The 17,265 fecal samples in our study 
123 were collected from baboons who ranged in age from 7.4 months to 27.7 years, spanning these

124 animals' natural lifespans (fig. S1A). Each baboon was sampled a median of 19 times, and 124

125 baboons were sampled at least 50 times (fig. S1B). On average, these samples spanned 4.3 years

126 of a baboon's life (range = 4 days to 13.2 years; fig. S1C), with a median of 35 days between

127 consecutive samples (fig. S1D).

128 A large majority of the microbiome samples we use here were published in Grieneisen et

129 al. (24), but we include 1,031 additional samples that were generated at the same time using the

130 same methods (they were not included in Grieneisen et al. (24) because we lack pedigree

131 information for these hosts). Briefly, we generated 896,911,162 sequencing reads (mean =

$13251,913.6$ reads per sample; range $=1021-477,241$, fig. S1E). We retained microbial amplicon

133 sequence variants (ASVs) with a minimum of 3 reads per sample that were seen in at least $20 \%$

134 of the samples, resulting in 341 microbial taxa at the ASV level (mean = 162 ASVs per sample;

135 range = 19 - 311 ASVs; fig S1F). DNA concentration and ASV diversity were not predicted by

136 time since sample collection (fig. S1G, S1H). Read counts were centered log-ratio transformed

137 prior to all subsequent analyses $(33,34)$. 
A

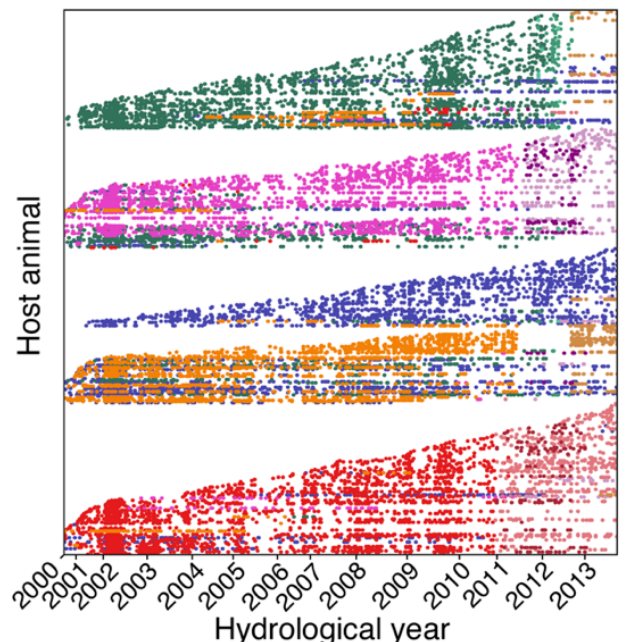

\section{Social groups}

- Weaver's (2000 - 2012) Kelly's $(2012-2012)$ Laza's (2012 - 2012) Linda's (2000 - 2011) Narasha's (2011 - 2013) Mica's $(2011-2013)$ Viola's (2000 - 2013) Omo's (2000 - 2011) Acacia's (2012 - 2013) Nyayo's $(2000-2011)$ Hokey's (2010 - 2013)

Snap's (2010 - 2012)
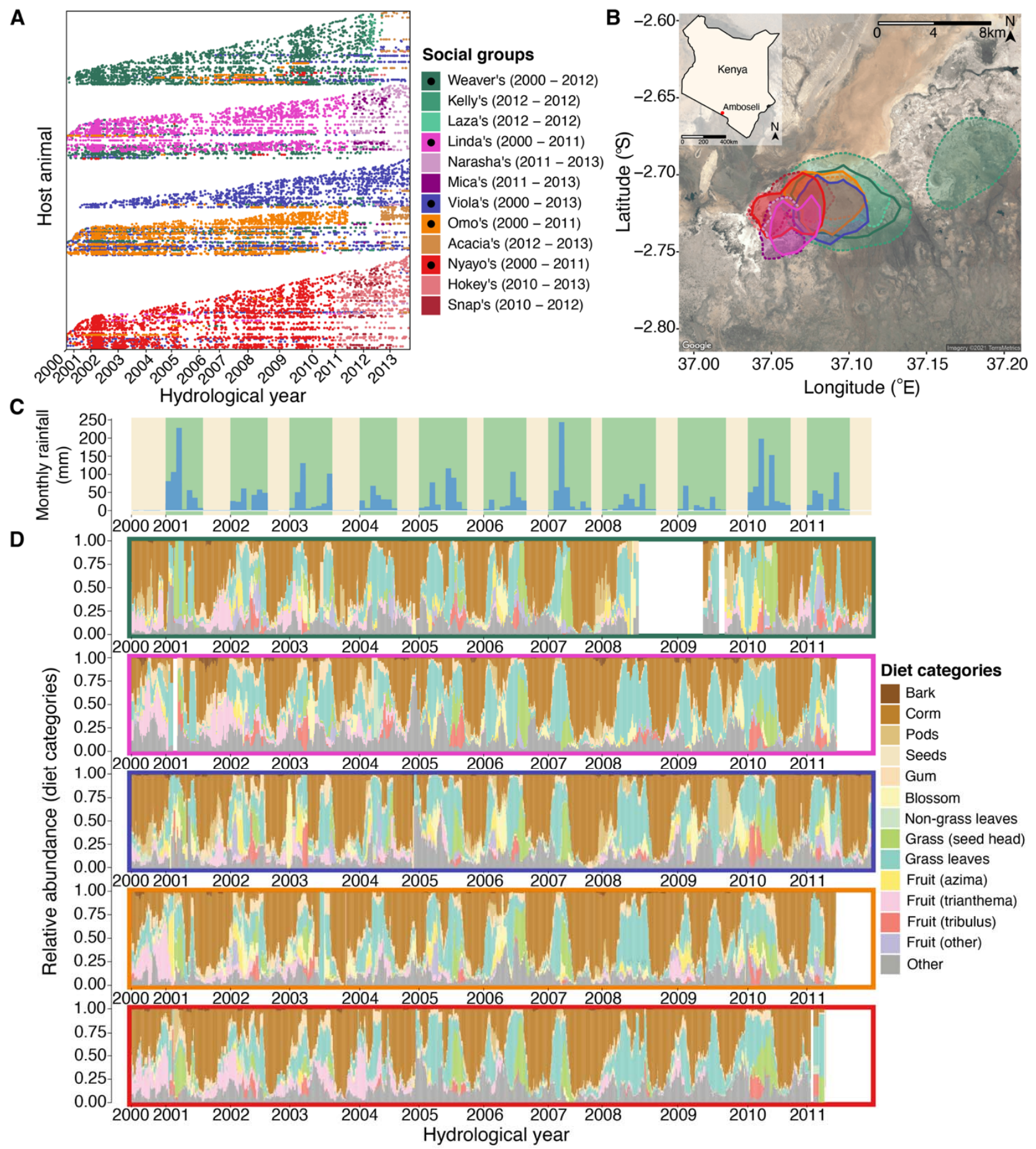

139 Fig. 1. Baboons in Amboseli experience shared environments at multiple scales. (A) The

140 microbiome time series consisted of 17,265 16S rRNA gut microbiome profiles. Each point

141 represents a microbiome sample, plotted by the date it was collected (x-axis). Each row (y-axis)

142 corresponds to a unique individual host. Samples were collected from 600 wild baboons living in

1435 original social groups (indicated by dark colors marked with black dots in the legend) and 7 
144 groups that fissioned/fused from these original groups (no black dots). (B) All baboon groups

145 ranged over a shared $\sim 60 \mathrm{~km}^{2}$ area, and the social groups had largely overlapping home ranges.

146 Ranges are shown as 90\% kernel densities over the sampling period specific to each group; 5

147 original social groups are shown with solid borders, fission and fusion products with dashed

148 borders. (C) Monthly rainfall amounts (blue bars, in $\mathrm{mm}$ ) with yellow and green stripes

149 representing dry and wet seasons, respectively, with the width reflecting the number of months

150 within the focal year that had at least $1 \mathrm{~mm}$ rainfall. (D) Temporal shifts in diet from the years

$1512000-2013$, shown as the relative abundance of diet components in the 5 original social groups

152 over 30-day sliding windows. Colors correspond to the 13 most common food types, while the

153 grey bars correspond to other or unknown food types. Colored boxes around each panel reflect

154 each of the 5 original, most extensively sampled social groups (colors as in plots A and B). The

155 white bars indicate time periods where no diet data were collected.

To test whether shared environmental conditions and host traits lead to similar gut

158 microbial compositions and synchronized dynamics across the microbiome metacommunity, we

159 used three main approaches (see Supplementary Materials for details of all analyses). First, we

160 characterized patterns of temporal autocorrelation to identify hallmarks of compositional

161 similarity and synchrony over time. Our expectation was that, if different baboons exhibit similar

162 gut microbiome compositions and synchronized microbiome dynamics, then samples collected

163 close in time across the metacommunity should be compositionally similar, and samples

164 collected from the same host should not be substantially more similar than samples from

165 different baboons. Alternatively, if hosts or social groups exhibit idiosyncratic compositions and

166 dynamics, then samples collected close in time from the same baboon, or the same group, should

167 be much more similar than they are to samples collected from different baboons living in

168 different groups. These analyses were run in $\mathrm{R}(\mathrm{v}$ 4.0.2; (35)) using custom-written functions

169 (code and analyzed data are available on GitHub/OSF; see Data Statement).

170 Second, to test whether dispersal limitation could explain microbiome idiosyncrasy, we

171 estimated metacommunity-wide microbial migration probabilities in each season and year using

172 the Sloan Neutral Community Model for Prokaryotes $(36,37)$. This model assumes that each

173 local community, defined as the microbial composition of a single host in a given season-year 
174 combination, is the outcome of stochastic population dynamics and microbial immigration from

175 other hosts in the microbiome metacommunity (i.e., other local communities). Briefly, local

176 communities have a constant size $N$, and individual microbes within each local community die at

177 a constant rate. These deaths create vacancies that can be occupied, either by individuals

178 immigrating from the microbiome metacommunity (with probability $m$ ), or by the offspring from

179 any taxon within the local community (i.e., from reproduction within the same host, with

180 probability $1-m)$. Species that are common in the metacommunity have a higher chance of

181 occupying vacancies than rare species. Without immigration from the microbiome

182 metacommunity, ecological drift leads each host's microbial diversity to reduce to a single taxon.

183 Thus, the migration probability, $m$, represents the metacommunity-wide probability that any

184 taxon, randomly lost from a given host/local community, will be replaced by dispersal from the

185 microbiome metacommunity, as opposed to reproduction within hosts (36, 37). Following Burns

186 et al. (38), $m$ can be interpreted as a measure of dispersal limitation, such that low migration

187 probabilities signify high dispersal limitation. We estimated season and hydrological year-

188 specific values for $m$ by defining the microbiome metacommunity as either the hosts' social

189 group or the whole host population. We fit neutral models using nonlinear least-squares

190 regression as implemented in the $\mathrm{R}$ package tyRa (39).

191 Third, to quantify the relative magnitude of idiosyncratic versus synchronized gut

192 microbiome dynamics for different microbiome features, we used generalized additive models

193 (GAMs) to capture non-linear, longitudinal changes in 52 gut microbiome features, including

194 three principal components of microbial community variation, three indices of alpha diversity

195 (species richness, the exponent of Shannon's H, and the inverse Simpson index, as computed by

196 the function reyni from the R package vegan (40)), and the relative abundances of all 12 phyla

197 and 34 families present in our data set, post filtering. GAMs allowed us to calculate the percent

198 deviance in each feature's dynamics attributable to factors that could contribute to synchronized

199 dynamics at different scales; percent deviance is a measure of goodness-of-fit for nonlinear

200 models and is analogous to the unadjusted $\mathrm{R}^{2}$ for linear models. We considered three scales:

201 factors experienced by the whole host population (e.g., rainfall and temperature), those

202 differentiated by social groups (e.g., group identity, group home range location, and diet), and

203 those differentiated at the level of individual hosts (e.g., host identity, sex, age, and social

204 dominance rank; see below for complete model structures). If shared environments and traits 
205 synchronize gut microbiome dynamics across hosts, these factors should explain substantial

206 deviance in microbiome dynamics. Alternatively, if microbiome dynamics are idiosyncratic,

207 population- and group-level factors will not explain considerable deviance and, instead, a large

208 fraction of the deviance will be attributable to host identity, controlling for shared environments,

209 behaviors, and traits. To ensure sufficiently dense sampling for identifying host- and group-level

210 dynamics, all three models were run on a subset of the full data set, consisting of 4,277 16S

211 rRNA gene sequencing profiles from the 56 best-sampled baboons living in the 5 social groups

212 sampled the longest (between 2002 and 2010; $\min =48$; median = 72.5; $\max =164$ samples; fig.

213 S2). GAMs were fit using the R package mgcv (41-43).

214 Notably, the GAM approach allows us to identify the percent deviance attributable to

215 host identity, but does not identify the specific characteristics that account for host identity

216 effects. Genetic effects are a likely candidate, as previous analyses demonstrate that taxon

217 abundance and summaries of gut microbiome position are lowly to moderately heritable in this

218 population (24). To evaluate this possibility, we tested the relationship between the deviance

219 explained in our GAMs for each microbiome taxon and the heritability of that taxon's relative

220 abundance (24). If host effects on microbiome dynamics are in part explained by host genotype,

221 we predicted that taxon heritability should be positively correlated with deviance explained at the

222 host level (i.e., model $\mathrm{P}+\mathrm{G}+\mathrm{H}$ ), but not at the group or population level (i.e., model P and model

$223 \mathrm{P}+\mathrm{G})$. 


\section{Results and Discussion}

225 Baboon gut microbiota exhibit cyclical shifts in community composition across seasons and 226 years

We began by visualizing annual and inter-annual fluctuations across the gut microbiome metacommunity over the 14-year span of the data. Consistent with prior research on primates (44-46), we found population-wide, cyclical shifts in microbiome composition across seasons and years (Fig. 2). This wet-dry seasonal cyclicity was primarily observable in the first principal component (PC1) of a principal component analysis (PCA) of clr-transformed read counts for all

232 17,265 samples (Fig. 2A, 2B; fig. S3-S5; PC1 explains 16.5\% of the variance in microbiome

233 community composition). PC1 tended to exhibit low values during the dry season, and high

234 values during the wet season, mirroring monthly rainfall (Fig. 2B; fig. S5). PC1 was also linked

235 to annual rainfall across years, exhibiting especially low values throughout 2008 and 2009,

236 which corresponded to the worst continuous drought in the Amboseli ecosystem in 50 years (Fig.

237 2A, 2B). We also observed small, but statistically significant seasonal differences in PC2 and

238 PC3 (8.4\% and 3.7\% of variation in community composition; Fig. 2C; fig. S3-S5) and in measures of alpha diversity (Fig. 2C; fig. S5, S6), as has been reported in other ecosystems (47).

In terms of individual microbiome taxa, $17 \%$ of phyla ( 2 of 12) and $38 \%$ of families (13 of 34) exhibited significant changes in relative abundance between the wet and dry seasons (Fig.

242 2C; table S1; linear models with a false discovery rate (FDR) threshold $=0.05$ for $\mathrm{n}=393$

243 models). These changes were significant for the phyla Firmicutes and Tenericutes (Fig. 2C, 2D;

244 fig. S7), and were especially pronounced for the families Helicobacteraceae, Coriobacteriaceae,

245 Burkholderaceae, Bacteroidales RF16 group, vadinBE97, Spirochaetaceae, and

246 Campylobacteraceae (Fig. 2C; fig. S8). 28\% of ASVs also exhibited significant changes in

247 abundance across seasons (97 of 341 ASVs; linear models with FDR threshold $=0.05$ for $\mathrm{n}=$

248393 models; fig. S9; table S2). The majority of gut microbial taxa at the ASV, family and

249 phylum level did not exhibit significant changes in abundance across seasons, suggesting that

250 these taxa play consistent roles in the gut ecosystem throughout the year, including

251 Kiritimatiellaeota, Elusomicrobia, Ruminococcacaceae, Clostridiaceae 1, and Rikenellaceae

252 (Fig. 2C; fig. S7, S8; table S1). 

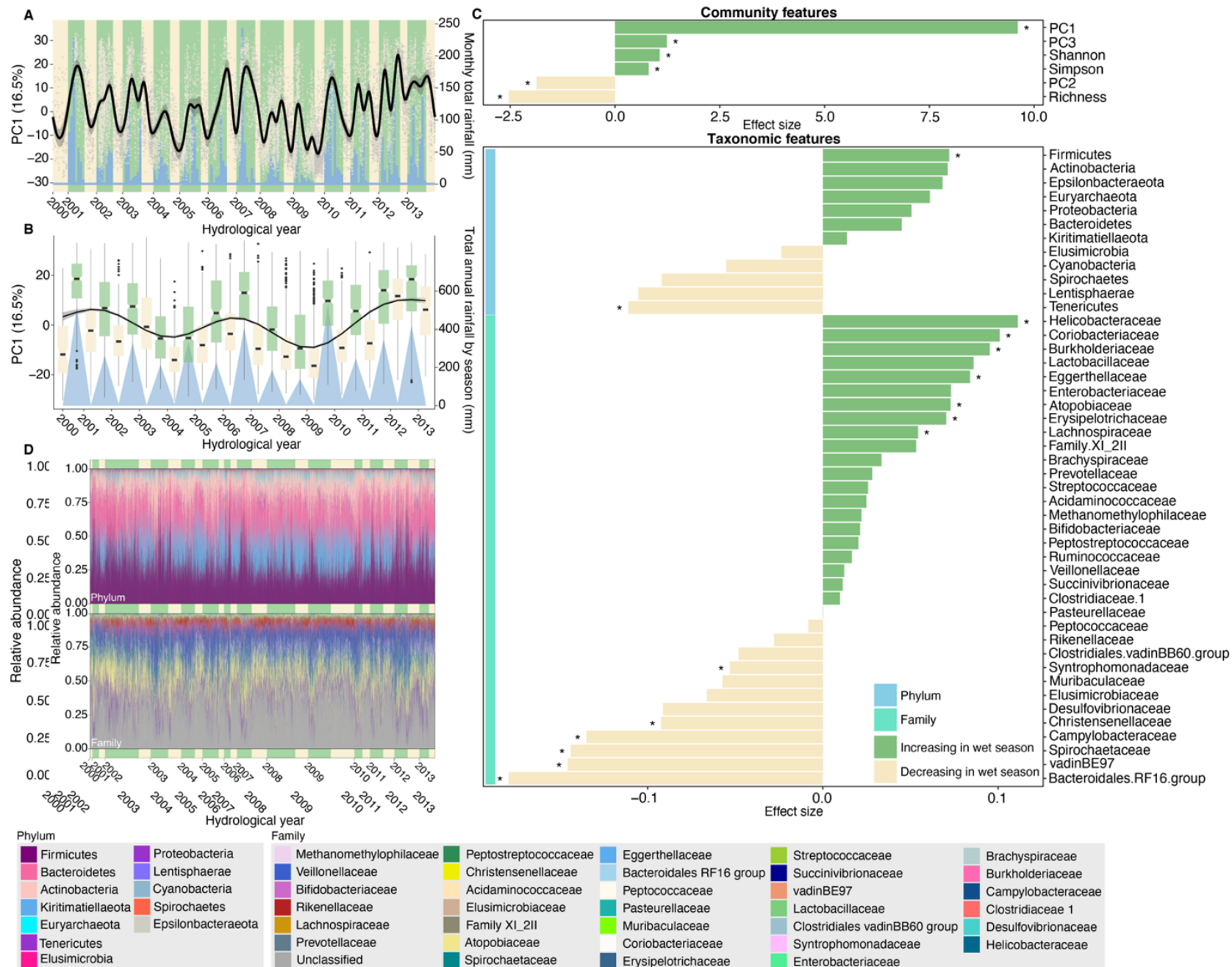

254 Fig. 2. Baboons show population-wide, cyclical shifts in microbiome composition across

seasons and years. (A) Changes in microbiome PC1 mirror monthly rainfall across the 14 years of the data set. The grey points show each sample's value for PC1 (y-axis) on the date it was collected. The black line shows the predicted daily trend for PC1 across samples, treating time

258 (x-axis) as a continuous variable from April 21, 2000 to September 19, 2013. The corresponding 259 gray ribbon shows the 95\% simultaneous confidence interval. Blue bars show monthly rainfall

260 (right-hand y-axis). Yellow and green bars in the background represent dry and wet seasons,

261 respectively, with the width reflecting the number of months within the focal year with at least 1

262 mm rainfall. (B) Changes in microbiome PC1 on an annual scale across the 14 years of the data

263 set. The box plots show the average distribution of microbiome PC1 in wet (green) and dry

264 (yellow) seasons. The black line shows the estimated annual trend for PC1 across all

265 hydrological years, and the blue triangles show total annual rainfall (right-hand y-axis). (C) The 
effect of season varies across 52 features of the microbiome, including six community features (top panel) and 46 taxonomic features (bottom panel; 12 phyla: light blue vertical bar; 34 families: turquoise vertical bar; for 341 ASVs, see Fig. S12). Each horizontal bar shows the effect of season from linear mixed models, with each feature as the dependent variable. Asterisks indicate features that changed significantly between the wet and dry seasons (FDR threshold $=$

2710.05 for $\mathrm{n}=393$ models). See figs. S7, S8 for feature-specific smooths and fig. S9 and table S2

272 for results for ASVs. Samples from the same host collected on the same date were averaged prior

273 to running the linear models. (D) Bar plots showing the relative abundance of all 12 microbial

274 phyla (above) and 34 families (below) across all samples. Green and yellow bars in the

275 background represent wet and dry seasons, with the width corresponding to the number of 276 samples in the focal hydrological year and season.

\section{Baboons exhibit largely idiosyncratic gut microbiome compositions and dynamics}

While the microbiome metacommunity exhibited cyclical, seasonal shifts in

280 composition, microbiome dynamics across different baboons were not strongly synchronized.

281 Instead, patterns of temporal autocorrelation indicated that each baboon exhibited largely

282 individualized gut microbiome compositions and dynamics (Fig. 3). In support, samples

283 collected from the same baboon within a few days were much more similar to each other than

284 they were to samples collected from different baboons over the same time span, regardless of

285 whether those animals lived in the same or a different social group (Fig 3A, 3B; Kruskal-Wallis:

$286 \mathrm{p}<2.2 \times 10^{-16}$ for all comparisons). Likewise, a PERMANOVA of Aitchison distances between all

287 samples revealed that host identity explained $8.6 \%(\mathrm{p}<0.001)$ of the variation in community

288 composition, much larger than sampling day or month $\left(\mathrm{r}^{2}=2.5 \%\right.$ and $\left.1.4 \%\right)$, group membership

289 (2.2\%), or the first three principal components of diet (0.04\% to 2.4\%; table S3; fig. S10).

Compositional similarity among samples from the same baboon fell steeply for samples

291 collected a few days to a few months apart (Fig. 3A, 3C). However, similarity rose again slightly

292 at 12-month intervals, reflecting the seasonal dynamics in Fig. 2. These 12-month peaks in

293 similarity were visible, even for samples collected more than 5 years apart, indicating that

294 individual hosts and the population at large return to somewhat similar microbiome community

295 states on 12-month cycles across years (Fig. 3C). Individualized host compositional signatures 
persisted for several years (Fig. 3A, 3C; fig. S11). Indeed, the 95\% confidence intervals on Aitchison similarity between samples collected from the same vs different hosts rarely overlapped for samples collected less than two years apart (fig. S11B, S11C). compositional similarity between hosts living in the population at the same time (Fig. 3D; fig.

301 S12). For instance, especially dense sampling during the 2008-2009 hydrological year meant that

302 we were able to collect at least one sample, for at least 10 months of the year, from 17 of our 303 study subjects. When we aligned these time series, we observed no shared pattern of change in 304 the top three principal components of microbiome composition across time beyond some overall seasonal patterns in PC1, nor did we see convergence to similar values within any given month (Fig. 3D). Consequently, the microbiome of each baboon took a different path over the ordination space over the same 1-year span (fig. S12). We found similar results for another dense sampling period in the 2007-2008 hydrological year (fig. S13).

Microbiome taxa varied in their contributions to individualized gut microbiome compositions (Fig. 3E; fig. S14). For example, for the 56 best-sampled hosts (fig. S2), several

311 phyla and families exhibited substantial variation in host mean (clr-transformed) relative

312 abundance (i.e., across repeated samples for that host) compared to their mean (clr-transformed)

313 relative abundance across all hosts. These taxa included members of the phyla Cyanobacteria,

314 Spirochaetes, Lentisphaerae, and Elusimicrobia, and the families Spirochaetaceae, vadinBE97,

315 Elusimicrobaceae, and Muribaculaceae (Fig. 3E; fig. S14). These highly variable taxa tended to

316 exhibit, on average, below-average abundance compared to less variable taxa that tended to

317 exhibit, on average, above-average abundance, indicating that idiosyncratic dynamics may be

318 more often linked to uncommon than common taxa (fig. S15).

To test whether individualized gut microbiome compositions and dynamics could be

320 explained by microbial dispersal limitation between hosts, we used the Sloan Neutral

321 Community Model for Prokaryotes to estimate metacommunity-wide migration probabilities, $m$,

322 for each season and hydrological year $(36,37)$. As described above, $m$ provides a measure of

323 dispersal limitation because it represents the probability that "vacancies" in a local community

324 (i.e. a host's microbiome) will be replaced by the process of dispersal from the microbiome

325 metacommunity (i.e. other hosts), as opposed to reproduction within a focal host's microbial 
326 community $(36,37)$. We found little evidence that dispersal limitation contributed to

327 idiosyncratic compositions and dynamics; the estimated probability that a given ASV lost from a

328 host's microbiota would be replaced by an ASV from another host in the population was nearly

$32940 \%$ (the average host population-wide across season and hydrological years $m=0.373$; range =

$330 \quad 0.332$ to 0.416 ; black points on fig. S16). These migration probabilities are generally lower than

331 those Sieber et al. (8) found for marine sponges sampled from the same coastal location (range of

$332 m$ across sponge species: $\min =0.36$; median=0.78; $\max =0.86$ ) but much higher than for mice and

333 nematodes, both in natural and laboratory populations (mice: $m_{\text {wild }}=0.11$ and $m_{\text {lab }}=0.18$;

334 nematode: $m_{\text {wild }}=0.03$ and $m_{\mathrm{lab}}=0.01$ ), indicating that dispersal limitation is relatively low for

335 baboon microbiota in Amboseli.

Interestingly, when we re-defined the microbiome metacommunity to be the host's social group, instead of the whole host population, migration probabilities were similar (average $m$ across groups $=0.355$; range $=0.347$ to 0.365 ; colored points on fig. S16). Hence, social group membership likely does not represent a large barrier to microbial colonization between baboons, as ASVs are widely shared across all members of the host population.

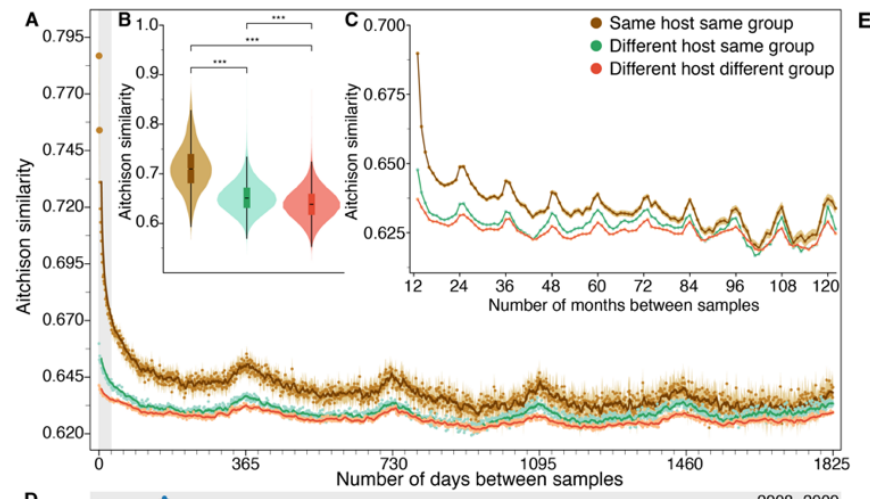

D

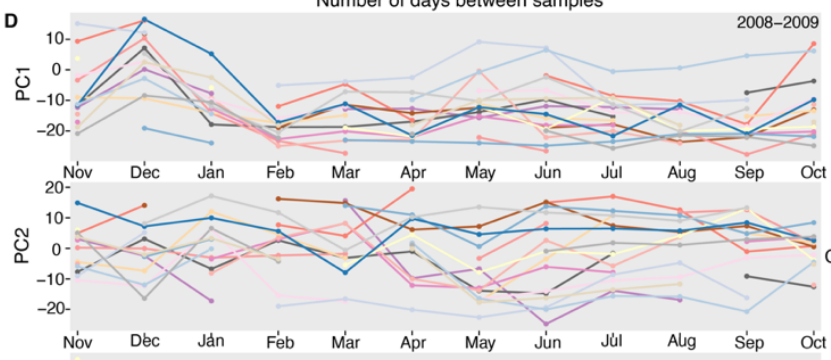

ช్

341

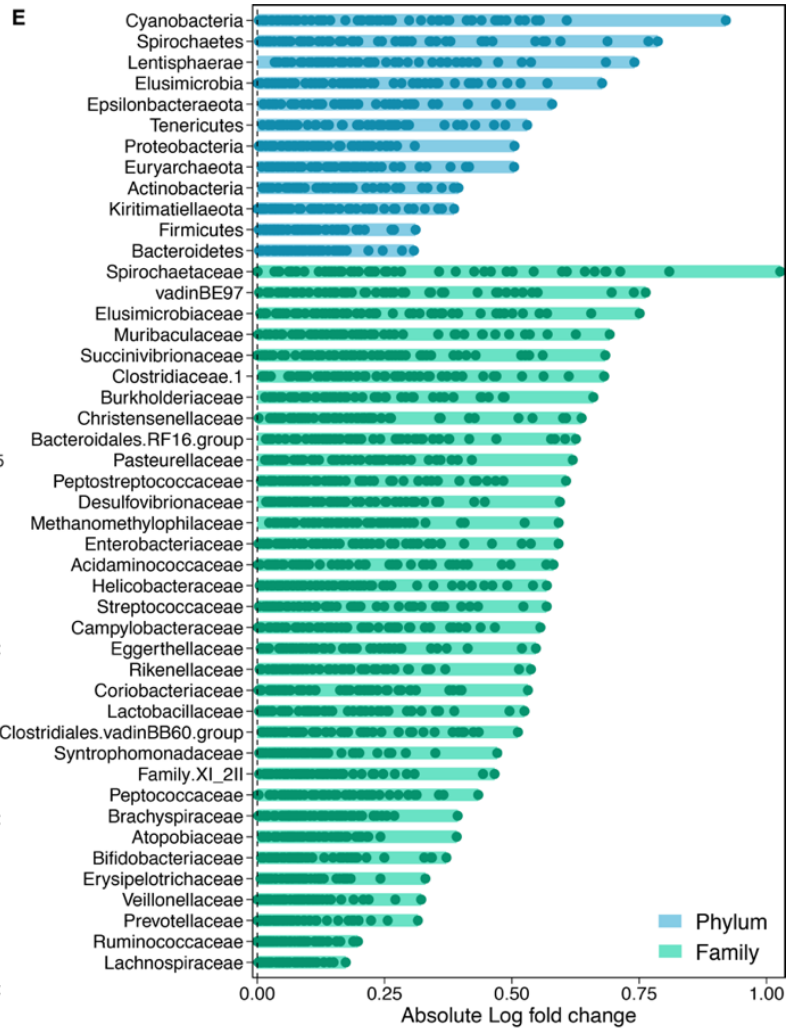




\section{Fig. 3. Baboons exhibit idiosyncratic gut microbiome compositions and dynamics. (A)}

343 Temporal autocorrelation in microbiome Aitchison similarity (y-axis) as a function of the time

344 between samples, plotted on a daily scale ( $\mathrm{x}$-axis), ranging from samples collected on the same

345 day to samples collected 5 years apart. Small tick marks correspond to months. Brown points

346 show average Aitchison similarity between samples collected from the same baboon; green

347 points show similarity between samples from different baboons living in the same social group;

348 orange points show similarity between samples from different baboons living in different social

349 groups. The lines represent moving averages (window size $=7$ days). (B) Average Aitchison

350 similarity among samples collected within 10 days of each other. Samples from the same baboon

351 are significantly more similar than samples collected from different baboons in the same or

352 different social groups (Kruskal-Wallis; $\mathrm{p}=2.22 \times 10^{-16}$ ). (C) Temporal autocorrelation in

353 microbiome Aitchison similarity on monthly scales for samples collected up to 10 years apart.

354 (D) Microbiome dynamics for 17 baboons for which we had at least one sample from 10 of the

35512 months of the 2008-2009 hydrological year (Nov 2008 to Oct 2009). Panels show each

356 individual's values for microbiome PC1, PC2, and PC3; each colored line represents a distinct

357 host. See fig. S13 for similar results during another densely sampled time period. Gaps indicate

358 that the focal host did not have a sample in a given month. (E) Some taxa have more

359 idiosyncratic abundances than others. Each horizontal bar shows a given taxon's minimum and

360 maximum absolute log fold change in abundance across the 56 best-sampled hosts (hosts are

361 represented as points within the bars; see fig. S2 for information on the best-sampled hosts).

362 Absolute fold changes were calculated, for a given taxon in a given host, as the taxon's average

363 clr-transformed abundance across all samples from that host, relative to the taxon's grand mean

364 in all hosts in the population. Hosts with large absolute fold changes for a given taxon therefore

365 have abundances of that taxon that are either well above or below-average compared to its

366 abundance in the host population at large (hosts with points close to zero exhibited taxonomic

367 abundances typical of the population at large). For many taxa, hosts varied in their absolute log

368 ratio values, indicating that they also deviated substantially from each other in the abundance of

369 those taxa. Taxa (y-axis) are ordered (from top to bottom) by their highest absolute log ratio

370 value across the 56 best-sampled hosts. Blue bars represent microbial phyla; green bars represent

371 families. See fig. S14 for a longitudinal version of this analysis for the most and least

372 idiosyncratic phyla and families. 


\section{Shared environmental conditions are linked to modest synchrony across hosts}

To quantify the relative magnitude of idiosyncratic versus synchronized gut microbiome

376 dynamics across the host population, social groups, and individual hosts, and to test whether

377 synchrony varies for different microbiome features, we used generalized additive models

378 (GAMs) to capture the nonlinear, longitudinal changes in 52 microbiome features (3 PCs of

379 community variation, 3 metrics of alpha diversity, and clr-transformed relative abundances of 12

380 phyla and 34 families). For each feature, we ran three GAMs to measure the deviance explained

381 in gut microbiome dynamics by successive sets of parameters, reflecting the nested nature of our

382 variables (Fig. 4A; x-axis of Fig. 4C; table S4). The population-level model (i.e., model P)

383 captured factors experienced by the whole host population, including average rainfall and

384 maximum daily temperature in the 30 days before sample collection and random effect splines to

385 capture monthly and annual cyclicity in microbiome features (e.g., Fig. 2A and B). The group-

386 level model (i.e., model $\mathrm{P}+\mathrm{G}$ ) included all the predictor variables in model $\mathrm{P}$, and added a

387 random effect spline for each social group, as well as variables to capture temporal changes in

388 each group's diet, home range use, and group size (Fig. 4A, 4C). The host-level model (i.e.,

389 model $\mathrm{P}+\mathrm{G}+\mathrm{H}$ ) included all of the predictor variables in model $\mathrm{P}+\mathrm{G}$, and added a random effect

390 spline for each host, and variables for host traits, including sex, age, and social dominance rank

391 (Fig. 4A, 4C). 


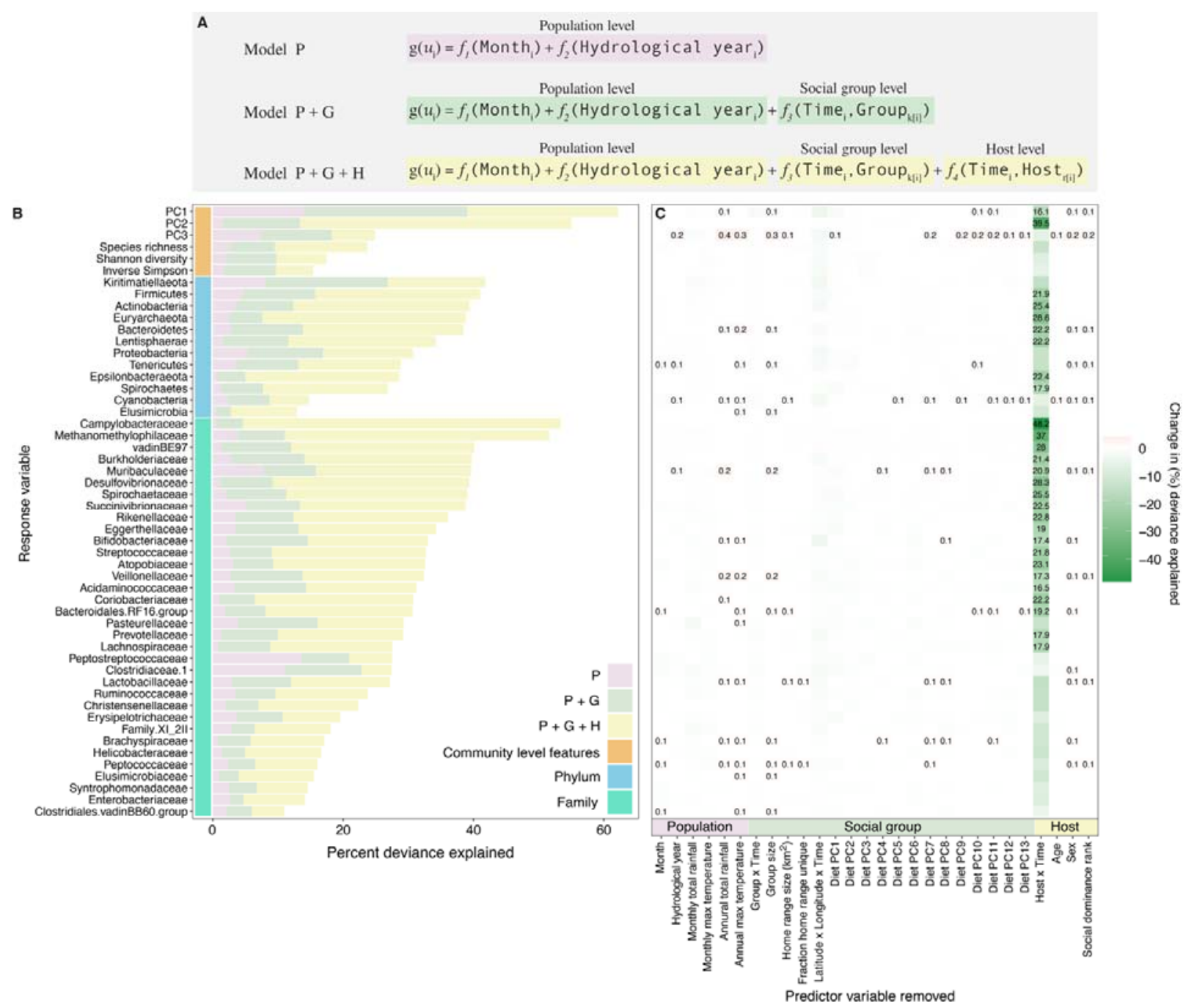

393 Fig. 4. Multilevel modeling identifies idiosyncratic dynamics. (A) We fit three hierarchical

394 GAMs to 52 microbiome features measured in 4,277 samples from the 56 best-sampled baboons

395 living in the 5 social groups sampled the longest (between 2002 and 2010; $\min =48$; median =

$39672.5 ; \max =164$ samples; fig. S2). Each model contained successive sets of predictor variables

397 reflecting population-level factors (pink), group-level factors (green) and host-level factors

398 (yellow). The factors at each level are listed at the bottom of panel C and defined in table S4).

399 Panel (B) shows for each microbiome feature (i.e., response variable), the deviance explained by

400 model $\mathrm{P}$ and the successive sets of predictor variables added in model $\mathrm{P}+\mathrm{G}$ and model $\mathrm{P}+\mathrm{G}+\mathrm{H}$,

401 respectively (table S5). Panel (C) shows the loss in deviance explained for model $\mathrm{P}+\mathrm{G}+\mathrm{H}$ as we

402 successively removed each predictor variable in turn from model $\mathrm{P}+\mathrm{G}+\mathrm{H}$, keeping the model

403 otherwise intact (table S6). Losses in deviance are shown in green, and we only provide numeric 
404 values for losses in deviance > 15\%. Gains in deviance are shown in red; we only show numeric 405 values for gains $>0.1 \%$.

Consistent with our autocorrelation analyses (Fig. 3), comparing the deviance explained for each microbiome feature across the three models revealed primarily idiosyncratic dynamics

409 for most microbiome features (Fig. 4B, 4C). Specifically, model P only explained on average

$4103.3 \%$ (range $=0.46 \%$ to $14.0 \%$ ) of the deviance across all 52 microbiome features (pink bars in

411 Fig 4B; table S5), compared to $8.1 \%$ on average for adding group-level factors to the

412 population-level model (increase from model $\mathrm{P}$ to model $\mathrm{P}+\mathrm{G}$; range $=2 \%$ to $25 \%$; green bars in

413 Fig. 4B; table S5), and 30.1\% of the deviance for including host-level dynamics (model P+G+H;

414 range $=11.0 \%$ to $62.2 \%$ ) in the same set of features (yellow bars in Fig. 4B; table S5).

415 Importantly, the added deviance for model $\mathrm{P}+\mathrm{G}+\mathrm{H}$ compared to model $\mathrm{P}$ or model $\mathrm{P}+\mathrm{G}$ was not

416 simply caused by including more parameters. Specifically, randomizing host identity and traits

417 across samples, while keeping each sample's annual, seasonal, and group identity intact, led to a

418 substantial drop in deviance explained relative to the real data (fig. S17). For instance, for PC2,

419 which captured the strongest host-level effects of all three PCs, the deviance explained by model

$420 \mathrm{P}+\mathrm{G}+\mathrm{H}$ dropped from $55 \%$ to $16.6 \%$ when host identity and traits were randomized (fig. S17;

421 see supplement and fig. S18 for an additional analysis investigating the effect of model

422 complexity on deviance explained). That said, for PC3, the addition of randomized host-level

423 dynamics still resulted in more than negligible deviance explained relative to the real data (3\% vs

$4246.6 \%$ ) suggesting that deviance explained may be inflated for some microbiome features.

44 of the 52 microbiome features exhibited greater gains in deviance explained by adding

426 host-level factors to model P+G, compared to adding group-level factors to model P, with 22

427 features gaining more than $20 \%$ deviance explained between model $\mathrm{P}+\mathrm{G}$ and model $\mathrm{P}+\mathrm{G}+\mathrm{H}$

428 (Fig. 4B; table S5). Three of the most common phyla, Actinobacteria, Bacteroidetes, and

429 Firmicutes all gained $>20 \%$ deviance explained between model $\mathrm{P}+\mathrm{G}$ and model $\mathrm{P}+\mathrm{G}+\mathrm{H}$

$430 \quad($ Actinobacteria $=27.1 \%$; Bacteroidetes $=24.6 \%$, and Firmicutes $=25.2 \%$; Fig. 4B; table S5).

431 The most idiosyncratic features (i.e., those that gained $>30 \%$ deviance explained by adding host-

432 level factors), were microbiome PC2, the phylum Euryarchaeota, and the families

433 Campylobacteraceae, Methanomethylophilaceae and Desulfovibrionaceae (Fig. 4B; table S5). 
434 Notably, even the most synchronous feature, microbiome PC1 (14\% deviance explained by the P 435 model), gained $23.2 \%$ deviance explained when adding host-level factors to the P+G model.

437 covariates intact, revealed that host identity explained nearly all of the deviance in our models

438 (Fig. 4C; table S5; average loss in deviance explained by removing host identity $=17.3 \%$

439 compared to $0.2 \%$ deviance for all other factors). Beyond host identity, the next most important

440 factor was the geographic area where the group had been travelling in the 30 days prior to

441 sample collection, which on average, explained 1\% of the deviance across all 52 features, with

442 the strongest effects on microbiome PC1, Bifidobacteraceae, and Kiritimatiellaeota (fig. S19;

443 table S5). The removal of all other individual predictor variables had only minor effects on

444 deviance explained (fig. S19; table S5).

445 To investigate whether some of the idiosyncrasy we observed, especially at the host level, 446 was due to genetic effects, we tested for a relationship between the deviance explained by each

447 GAM and the narrow-sense heritability $\left(h^{2}\right)$ of microbiome taxon abundance as estimated by

448 Grieneisen et al. (24). We found that higher levels of deviance explained by model $\mathrm{P}+\mathrm{G}+\mathrm{H}$ were

449 predicted by higher taxon heritability (Pearson correlation: $R=0.37, p=0.016$; Fig. 5A).

450 Reassuringly, we found no such effect at the population or group level, as expected since

451 genotype is a property of individual hosts, not groups or populations (model $\mathrm{P}+\mathrm{G}$ : $\mathrm{R}=0.047$,

$452 \mathrm{p}=0.76$; model P: R=0.0085, p=0.96; Fig. 5B). In particular, we explained substantially more

453 deviance by adding the host level to model $\mathrm{P}+\mathrm{G}$ for microbiome taxa with moderate to high $h^{2}$

454 values (i.e., those $>0.05$ ) than we did for taxa with low $h^{2}$ values (model $\mathrm{P}+\mathrm{G}+\mathrm{H}$ : $\min =16.0$,

455 median=32.6, $\max =53.4$ vs model $\mathrm{P}+\mathrm{G}$ : $\min =4.6$, median=11.1, $\max =26.8$; Fig. 5B ). These

456 results suggest that some idiosyncrasy in gut microbiome dynamics is a consequence of host

457 differences in genotype. We note, however, that because $h^{2}$ estimates from the animal model

458 cannot be mapped directly onto estimates of deviance explained in GAMs, direct estimates of

459 genetic versus environmental effects on host dynamics remain an important topic for future 460 work. 


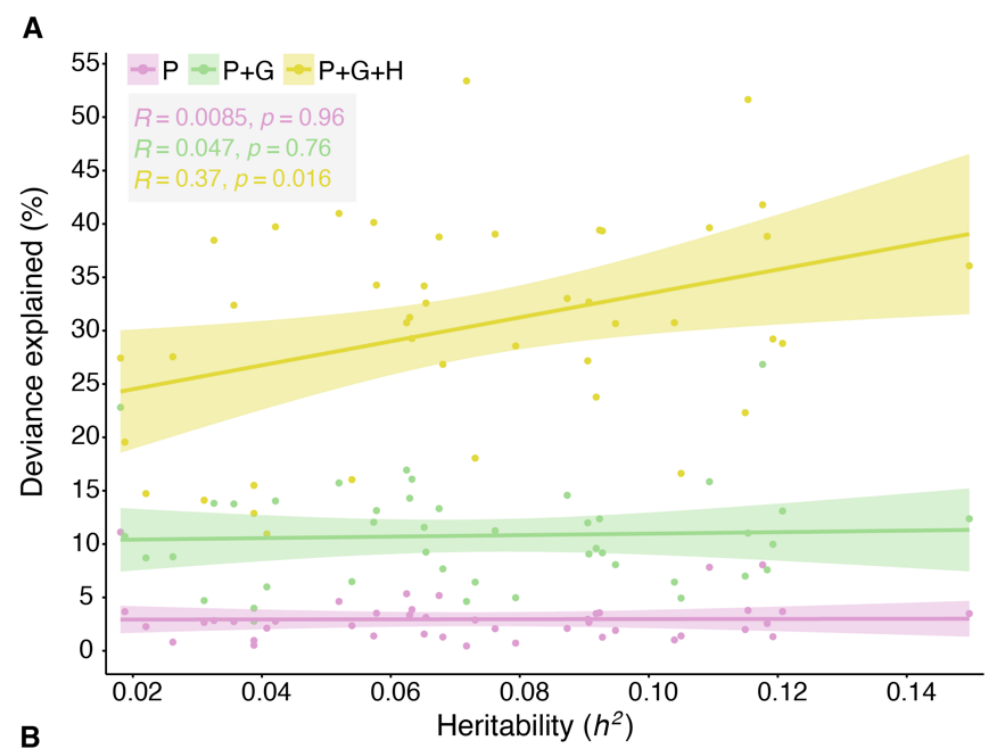

B

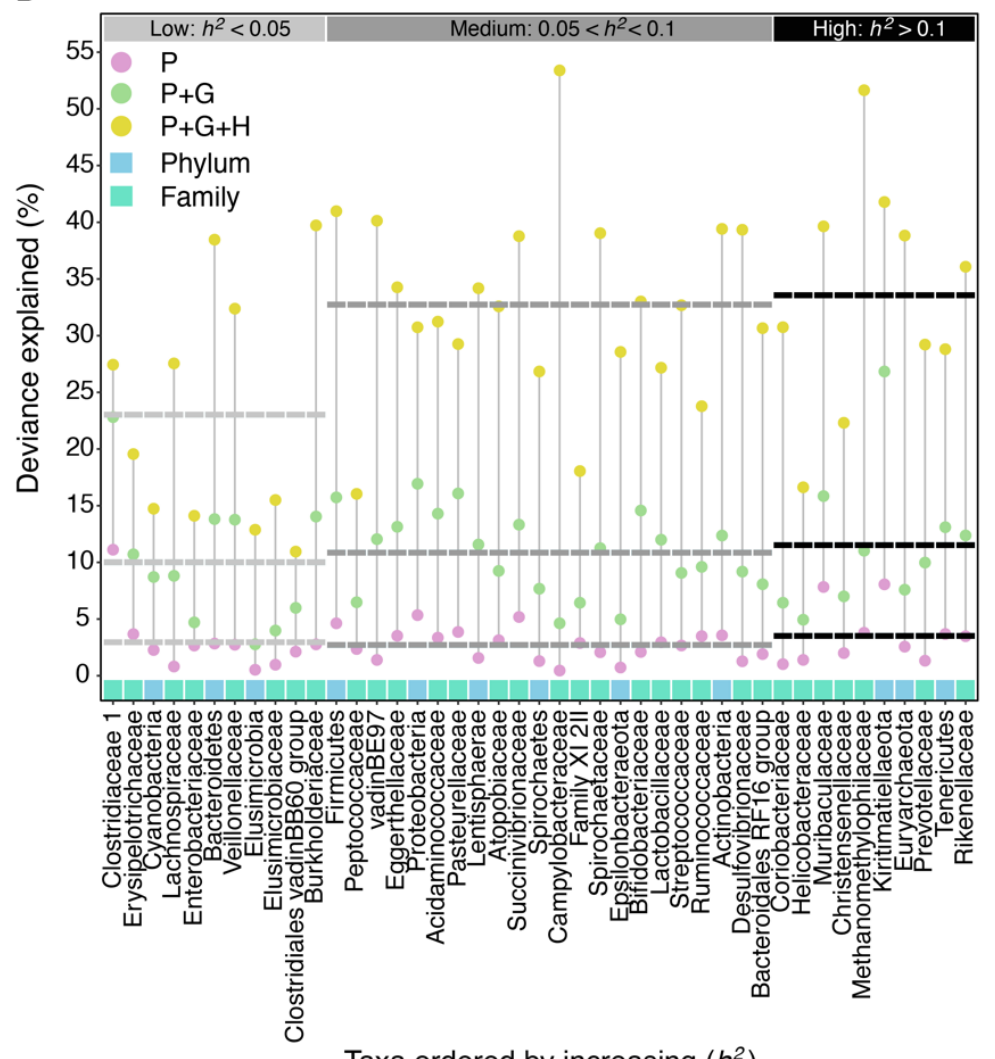

462 Fig. 5. Microbiome taxon heritability is associated with idiosyncratic dynamics. (A)

463 Deviance explained (y-axis) by the phylum and family level GAMs (from Fig. 4) plotted against

464 the focal taxon's heritability estimate $\left(h^{2}\right.$; x-axis). Pink, green and yellow denote model P, model

$465 \mathrm{P}+\mathrm{G}$ and model $\mathrm{P}+\mathrm{G}+\mathrm{H}$, respectively. (B) Deviance explained (y-axis) across the model

466 hierarchy (pink: model P; green: model P+G; yellow: model $\mathrm{P}+\mathrm{G}+\mathrm{H}$ ) for each taxonomic feature 
467 (i.e., at the phylum and family level; $\mathrm{x}$-axis). The $\mathrm{x}$-axis is ordered by increasing heritability with 468 light blue and turquoise squares representing phyla and families, respectively. Horizontal dashed 469 lines show the average deviance explained per model for taxa with low heritability estimates $\left(h^{2}\right.$ $470<0.05$; light gray); medium heritability estimates $\left(0.05<h^{2}<0.1\right.$; dark gray $)$; and high

471 heritability estimates $\left(h^{2} \geq 0.1\right.$; black).

473 Gut microbiome dynamics among social group members are more synchronized than for 474 the host population at large

475 Previous research in humans and other social mammals, including the Amboseli baboons, 476 finds that hosts in the same social group often have more similar gut microbiome compositions 477 than hosts in different social groups (e.g. 32, 48-50). Likewise, in our current data set, several 478 taxa exhibited abundances that were, on average, higher or lower within a given social group 479 compared to their average abundance in the host population at large (fig. S20, S21). Hence, we 480 tested whether shared social group membership is linked to greater microbiome synchrony than 481 hosts in different groups. In support, the patterns of temporal autocorrelation in Fig. 3A showed 482 that hosts in the same group have detectably more similar microbiomes than those in different 483 groups, especially for samples collected within 10 days of each other (Fig. 3B; Kruskal-Wallis: p

$\left.484<2.2 \times 10^{-16}\right)$. Likewise, samples from the same group tended to occupy similar ordination space 485 over time (video S2). While small, these group-level similarities were detectable, even for 486 samples collected more than 2 years apart (Fig. 3C; fig. S11A). The addition of group-level 487 splines to our GAMs led to gains in deviance that explained more than $10 \%$ for 15 of 52 488 microbiome features, including all three microbiome PCs, five phyla, and seven families (Fig. 489 4B, 4C; table S5). Several of these taxa were abundant in hosts, such as Firmicutes, 490 Bacteroidetes, and Bifidobacteriaceae (Fig. 4B, 4C; table S5).

491 Because each social group has a somewhat distinctive gut microbiota, the effects of 492 climate and diet on microbiome dynamics may differ across groups. To test this idea, we added 493 interaction effects between group identity and climate variables (rain and temperature), or 494 between group identity and the first three PCs of diet to model P+G+H. However, these 495 interactions did not lead to substantial gains in deviance explained in our models (fig. S22; table 496 S7). For instance, adding the climate interactions explained on average an additional $0.95 \%$ 
deviance across all 52 features (range $=-1.9 \%$ to $5.4 \%$; table S7), and diet interactions explained, on average, an additional $1.2 \%$ deviance across all 52 features (range $=-0.7 \%$ to $5.6 \%$; table S7).

Gut microbial congruence among group members could also be linked to shared behaviors and environments: baboons in the same group eat the same foods at the same time, travel as a unit across the landscape, and may be grooming partners that are frequently in physical contact (Fig. 1B, 1C; video S1; (24, 27-31)). Indeed, after host identity, the next most

504 important predictor variable in model $\mathrm{P}+\mathrm{G}+\mathrm{H}$ was the group's home range in the 30 days before 505 sample collection (fig. S19; table S6). Despite previous evidence for increased similarity in 506 microbiome profiles among grooming partners in the Amboseli baboons (32), we did not find

507 evidence for this pattern in our current data set (fig. S23). Indeed, samples collected within 30

508 days of each other from individuals with strong grooming bonds were not substantially more 509 similar than samples from animals with weak or no observed grooming relationship (mean 510 Aitchison similarity between pairs with strong bonds $=0.645$; mean Aitchison similarity between 511 pairs weak or no bond =0.646; fig. S24). Because of differences in methodology, the lack of a 512 grooming effect in this data set should be interpreted with caution. Our prior research on this 513 topic (32) characterized microbial communities using shotgun metagenomic sequencing from $514>90 \%$ of social network members, all within 30 days of each other. In contrast, this current data 515 set relies on 16S rRNA gene sequencing data from sparsely-sampled networks. Shotgun 516 metagenomic data provide much higher taxonomic resolution than 16S rRNA identities, and may 517 therefore more accurately capture the direct transmission between hosts.

\section{Conclusions}

We tested, for the first time, whether gut microbiome dynamics are synchronized among

521 hosts experiencing strong synchronizing forces, including shared environments, similar diets, 522 and high rates of between-host microbial dispersal. Despite these forces, baboons in Amboseli

523 exhibit largely idiosyncratic gut microbiome dynamics: samples from the same baboon collected

524 within a few days of each other were much more similar to each other than samples from

525 different baboons, and host-specific factors, especially host identity, collectively explained 30\% 526 of the deviance in microbiome dynamics, compared to just 3\% for factors shared across the host 
527 population. These idiosyncratic dynamics suggest that microbiome personalization is a

528 widespread phenomenon that is likely not unique to humans, and instead may be shared with

529 other social mammals. This microbiome personalization likely emerges from ecological and

530 evolutionary phenomena that are a normal part of complex microbial communities, such as

531 priority effects, functional redundancy, and horizontal gene flow. Together, these forces are

532 expected to lead microbes with the same taxonomic identity in different hosts to perform

533 somewhat different functions, experience different competitive landscapes and selective regimes,

534 and play different ecological roles $(15,16)$. As a result, the same microbial taxa may often

535 respond in different ways to environmental fluctuations, chance events, and/or interactions with

536 other microbes in different hosts, producing personalized, rather than synchronized dynamics.

537 This personalization means that microbiome research aimed at improving human and

538 animal health could face challenges to developing broadly applicable therapies, beyond those

539 caused by heterogeneity in host diets, behaviors, and environments. Microbiome researchers aim

540 to predict microbiome changes, link microbiome taxa and dynamics to health outcomes, and

541 design microbiome interventions that work well for large segments of the human population.

542 Personalization in humans is already presenting problems in attaining these goals. For instance,

543 predictive models of gut microbiome dynamics from one person have been shown to fail when

544 they are applied to other people (19). Our results suggest that microbiome predictions and

545 interventions focused on microbiome taxa will require approaches that are either personalized or

546 focus on microbial functions, as opposed to taxonomic identities. Even then, "universal"

547 microbiome therapies that work the same way for all hosts may be unattainable. Instead,

548 microbiome interventions will likely work best when they are designed for specific host groups

549 or populations that have shared compositions and dynamics. Further, we expect that the types of

550 prediction and intervention efforts that will suffer least from gut microbiome personalization are

551 those that focus on microbiome functional traits (e.g., metabolites; functional pathways), rather

552 than taxonomic composition. Together, our results provide novel insights about the extent and

553 ecological causes of microbiome personalization, and point towards ways to overcome these

554 barriers.

\section{Acknowledgments:}


557 We thank Jeanne Altmann for her essential role in stewarding the Amboseli Baboon Project, and

558 in collecting and maintaining the fecal samples used in this manuscript. We thank the Kenya

559 Wildlife Service, the National Council for Science, Technology, and Innovation, and the

560 National Environment Management Authority for permission to conduct research and collect

561 biological samples in Kenya. We also thank the University of Nairobi, Institute of Primate

562 Research, National Museums of Kenya, the Amboseli-Longido pastoralist communities, the

563 Enduimet Wildlife Management Area, Ker \& Downey Safaris, Air Kenya, and Safarilink for

564 their cooperation and assistance in the field. We thank Karl Pinc for managing and designing the

565 database. We also thank Tawni Voyles, Anne Dumaine, Yingying Zhang, Meghana Rao, Tauras

566 Vilgalys, Amanda Lea, Noah Snyder-Mackler, Paul Durst, Jay Zussman, Garrett Chavez, and

567 Reena Debray for contributing to fecal sample processing. Complete acknowledgments for the

568 ABRP can be found online at https://amboselibaboons.nd.edu/acknowledgements/.

569 Funding: This work was supported by the National Science Foundation and the National

570 Institutes of Health, especially NSF Rules of Life Award DEB 1840223 (EAA, JAG), and the

571 National Institute on Aging R21 AG055777 (EAA, RB) and NIH R01 AG053330 (EAA), and

572 NIH R35 GM128716 (RB), the Duke University Population Research Institute P2C-HD065563

573 (pilot to JT), the University of Notre Dame's Eck Institute for Global Health (EAA), and the

574 Notre Dame Environmental Change Initiative (EAA). Since 2000, long-term data collection in

575 Amboseli has been supported by NSF and NIH, including IOS 1456832 (SCA), IOS 1053461

576 (EAA), DEB 1405308 (JT), IOS 0919200 (SCA), DEB 0846286 (SCA), DEB 0846532 (SCA),

577 IBN 0322781 (SCA), IBN 0322613 (SCA), BCS 0323553 (SCA), BCS 0323596 (SCA),

578 P01AG031719 (SCA), R21AG049936 (JT, SCA), R03AG045459 (JT, SCA), R01AG034513

579 (SCA), R01HD088558 (JT), and P30AG024361 (SCA). We also thank Duke University,

580 Princeton University, the University of Notre Dame, the Chicago Zoological Society, the Max

581 Planck Institute for Demographic Research, the L.S.B. Leakey Foundation and the National

582 Geographic Society for support at various times over the years.

583 Author contributions: EAA, JRB, LBB, RB, JAG, SM, and JT designed the research; EAA,

584 SCA, RB, MRD, LG, JG, LRG, NG, SM, VY, NHL, TLW, RSM, JKW, LS, LBB, and JT,

585 produced the data; JRB, TJG, DAWAMJ, LG, JCG performed the bioinformatics; JRB, KR, SM,

586 performed the statistical analyses. EAA and JRB wrote the manuscript with important

587 contributions from all authors. 
Competing interests: The authors declare no competing interests.

589 Data and materials availability: 16S rRNA gene sequences are deposited on EBI-ENA (project

590 ERP119849) and Qiita [study 12949, (51)]. Analyzed data and code is available on the first

591 author's Open Science Framework / GitHub repository; for peer-review purposes, this is an

592 anonymized link: https://osf.io/erdxa/?view_only=3323f05a5a9b479bac1124a5b07a62a9 .

\section{References}

595 1. B. H. Schlomann, R. Parthasarathy, Timescales of gut microbiome dynamics. Curr Opin Microbiol 50, 56-63 (2019).

2. H. Koch, P. Schmid-Hempel, Socially transmitted gut microbiota protect bumble bees against an intestinal parasite. Proceedings of the National Academy of Sciences 108, 19288-19292 (2011).

3. C. T. Finnicum, J. J. Beck, C. V. Dolan, C. Davis, G. Willemsen, E. A. Ehli, D. I.
Boomsma, G. E. Davies, E. J. C. de Geus, Cohabitation is associated with a greater resemblance in gut microbiota which can impact cardiometabolic and inflammatory risk. BMC Microbiology 19, 230 (2019).

4. A. Bashan, T. E. Gibson, J. Friedman, V. J. Carey, S. T. Weiss, E. L. Hohmann, Y. Y. Liu, Universality of human microbial dynamics. Nature 534, 259-262 (2016).

5. E. K. Costello, K. Stagaman, L. Dethlefsen, B. J. Bohannan, D. A. Relman, The application of ecological theory toward an understanding of the human microbiome. Science 336, 1255-1262 (2012).

6. $\quad$ E. T. Miller, R. Svanback, B. J. Bohannan, Microbiomes as metacommunities: understanding host-associated microbes through metacommunity ecology. Trends in Ecology \& Evolution, (2018).

613

\section{J. Bjork, C. Díez-Vives, C. Astudillo-García, E. A. Archie, J. M. Montoya, Vertical} transmission of sponge microbiota is inconsistent and unfaithful. Nature Ecology \& Evolution 3, 1172-1183 (2019).

8. M. Sieber, L. Pita, N. Weiland-Brauer, P. Dirksen, J. Wang, B. Mortzfeld, S. Franzenburg, R. A. Schmitz, J. F. Baines, S. Fraune, U. Hentschel, H. Schulenburg, T. C. G. Bosch, A. Traulsen, Neutrality in the Metaorganism. PLoS Biol 17, e3000298 (2019).

9. A. T. Tredennick, C. dr Mazancourt, M. Loreau, P. B. Adler, Environmental responses, not species interactions, determine synchrony of dominant species in semiarid grasslands. Ecology 98, 971-981 (2017).

10. M. Loreau, C. de Mazancourt, Species synchrony and its drivers: neutral and nonneutral community dynamics in fluctuating environments. American Naturalist 172, E48-66 (2008).

11. F. I. Isbell, H. W. Polley, B. J. Wilsey, Biodiversity, productivity and the temporal stability of productivity: patterns and processes. Ecol Lett 12, 443-451 (2009).

12. A. Hector, Y. Hautier, P. Saner, L. Wacker, R. Bagchi, J. Joshi, M. Scherer-Lorenzen, E. M. Spehn, E. Bazeley-White, M. Weilenmann, M. C. Caldeira, P. G. Dimitrakopoulos, J. A. Finn, K. Huss-Danell, A. Jumpponen, C. P. H. Mulder, C. Palmborg, J. S. Pereira, A. S. D. Siamantziouras, A. C. Terry, A. Y. Troumbis, B. Schmid, M. Loreau, General 
stabilizing effects of plant diversity on grassland productivity through population asynchrony and overyielding. Ecology 91, 2213-2220 (2010).

13. C. de Mazancourt, F. Isbell, A. Larocque, F. Berendse, E. De Luca, J. B. Grace, B. Haegeman, H. W. Polley, C. Roscher, B. Schmid, D. Tilman, J. van Ruijven, A. Weigelt, B. J. Wilsey, M. Loreau, Predicting ecosystem stability from community composition and biodiversity. Ecology Letters 16, 617-625 (2013).

14. K. Gross, B. J. Cardinale, J. W. Fox, A. Gonzalez, M. Loreau, H. W. Polley, P. B. Reich, J. van Ruijven, Species richness and the temporal stability of biomass production: a new analysis of recent biodiversity experiments. Am Nat 183, 1-12 (2014).

15. S. Louca, M. F. Polz, F. Mazel, M. B. N. Albright, J. A. Huber, M. I. O'Connor, M. Ackermann, A. S. Hahn, D. S. Srivastava, S. A. Crowe, M. Doebeli, L. W. Parfrey, Function and functional redundancy in microbial systems. Nat Ecol Evol 2, 936-943 (2018).

16. P. B. Rainey, S. D. Quistad, Toward a dynamical understanding of microbial communities. Philos Trans R Soc Lond B Biol Sci 375, 20190248 (2020).

17. G. E. Flores, J. G. Caporaso, J. B. Henley, J. R. Rideout, D. Domogala, J. Chase, J. W. Leff, Y. Vazquez-Baeza, A. Gonzalez, R. Knight, R. R. Dunn, N. Fierer, Temporal variability is a personalized feature of the human microbiome. Genome Biology 15, 531 (2014).

18. J. G. Caporaso, C. L. Lauber, E. K. Costello, D. Berg-Lyons, A. Gonzalez, J. Stombaugh, D. Knights, P. Gajer, J. Ravel, N. Fierer, J. I. Gordon, R. Knight, Moving pictures of the human microbiome. Genome Biology 12, R50 (2011).

19. A. J. Johnson, P. Vangay, G. A. Al-Ghalith, B. M. Hillmann, T. L. Ward, R. R. ShieldsCutler, A. D. Kim, A. K. Shmagel, A. N. Syed, S. Personalized Microbiome Class, J. Walter, R. Menon, K. Koecher, D. Knights, Daily Sampling Reveals Personalized DietMicrobiome Associations in Humans. Cell Host \& Microbe 25, 789-802 (2019).

20. D. Rothschild, O. Weissbrod, E. Barkan, A. Kurilshikov, T. Korem, D. Zeevi, P. I. Costea, A. Godneva, I. N. Kalka, N. Bar, S. Shilo, D. Lador, A. V. Vila, N. Zmora, M. Pevsner-Fischer, D. Israeli, N. Kosower, G. Malka, B. C. Wolf, T. Avnit-Sagi, M. LotanPompan, A. Weinberger, Z. Halpern, S. Carmi, J. Fu, C. Wijmenga, A. Zhernakova, E. Elinav, E. Segal, Environment dominates over host genetics in shaping human gut microbiota. Nature 555, 210-215 (2018).

21. G. Falony, M. Joossens, S. Vieira-Silva, J. Wang, Y. Darzi, K. Faust, A. Kurilshikov, M. J. Bonder, M. Valles-Colomer, D. Vandeputte, R. Y. Tito, S. Chaffron, L. Rymenans, C. Verspecht, L. De Sutter, G. Lima-Mendez, K. D'Hoe, K. Jonckheere, D. Homola, R. Garcia, E. F. Tigchelaar, L. Eeckhaudt, J. Fu, L. Henckaerts, A. Zhernakova, C. Wijmenga, J. Raes, Population-level analysis of gut microbiome variation. Science 352, 560-564 (2016).

22. A. Zhernakova, A. Kurilshikov, M. J. Bonder, E. F. Tigchelaar, M. Schirmer, T. Vatanen, Z. Mujagic, A. V. Vila, G. Falony, S. Vieira-Silva, J. Wang, F. Imhann, E. Brandsma, S. A. Jankipersadsing, M. Joossens, M. C. Cenit, P. Deelen, M. A. Swertz, R. K. Weersma, E. J. M. Feskens, M. G. Netea, D. Gevers, D. Jonkers, L. Franke, Y. S. Aulchenko, C. Huttenhower, J. Raes, M. H. Hofker, R. J. Xavier, C. Wijmenga, J. Y. Fu, L. C. Study, Population-based metagenomics analysis reveals markers for gut microbiome composition and diversity. Science 352, 565-569 (2016). 
23. S. C. Alberts, J. Altmann, "The Amboseli Baboon Research Project: Themes of continuity and change" in Long-term field studies of primates, P. Kappeler, D. P. Watts, Eds. (Springer Verlag, 2012), pp. 261-287.

24. L. Grieneisen, M. Dasari, T. J. Gould, J. R. Bjo $\square$ rk, J. C. Grenier, V. Yotova, D. Jansen, N. Gottel, J. B. Gordon, N. H. Learn, L. R. Gesquiere, T. L. Wango, R. S. Mututua, J. K. Warutere, I. L. Siod, J. A. Gilbert, L. B. Barreiro, S. C. Alberts, J. Tung, E. A. Archie, R. Blekhman, Gut microbiome heritability is nearly universal but environmentally contingent. Science 373, 181-186 (2021).

25. T. Ren, L. Grieneisen, S. C. Alberts, E. A. Archie, M. Wu, Development, diet, and dynamism: longitudinal and cross-sectional predictors of gut microbial communities in wild baboons. Environmental Microbiology 18, 1312-1325 (2016).

26. L. Grieneisen, M. J. Charpentier, J. Altmann, S. C. Alberts, R. Blekhman, J. Tung, E. A. Archie, Genes, geology, and germs: gut microbiota across a primate hybrid zone are explained by site soil properties, not host species. Proceedings of the Royal Society $\mathbf{2 8 6}$, 20190431 (2019).

27. J. B. Silk, Activities and feeding behavior of free-ranging pregnant baboons. International Journal of Primatology 8, 593-613 (1987).

28. S. A. Altmann, Foraging for Survival: Yearling Baboons in Africa (University of Chicago Press, Chicago, 1998).

29. A. M. Bronikowski, J. Altmann, Foraging in a variable environment: Weather patterns and the behavioral ecology of baboons. Behavioral Ecology and Sociobiology 39, 11-25 (1996).

30. P. Muruthi, J. Altmann, S. Altmann, Resource base, parity and reproductive condition affect females' feeding time and nutrient intake within and between groups of a baboon population. Oecologia 87, 467-472 (1991).

31. J. M. Shopland, Food quality, spatial deployment, and the intensity of feeding interference in yellow baboons (Papio cynocephalus). Behav Ecol Sociobiol 21, 149-156 (1987).

32. J. Tung, L. B. Barriero, M. B. Burns, J. C. Grenier, J. Lynch, L. E. Grieneisen, J. Altmann, S. C. Alberts, R. Blekhman, E. A. Archie, Social networks predict gut microbiome composition in wild baboons. eLife 4, e05224 (2015).

33. J. T. Morton, C. Marotz, A. Washburne, J. Silverman, L. S. Zaramela, A. Edlund, K. Zengler, R. Knight, Establishing microbial composition measurement standards with reference frames. Nat Commun 10, 2719 (2019).

34. G. B. Gloor, J. M. Macklaim, V. Pawlowsky-Glahn, J. J. Egozcue, Microbiome datasets are compositional: and this is not optional. Front Microbiol 8, 2224 (2017).

35. R. R Core Team, "R: A language and environment for statistical computing. (available at http://www.R-project.org/)" (2020).

36. W. T. Sloan, M. Lunn, S. Woodcock, I. M. Head, S. Nee, T. P. Curtis, Quantifying the roles of immigration and chance in shaping prokaryote community structure.

Environmental Microbiology 8, 732-740 (2006).

37. W. T. Sloan, S. Woodcock, M. Lunn, I. M. Head, T. P. Curtis, Modeling TaxaAbundance Distributions in Microbial Communities using Environmental Sequence Data. Microbial Ecology 53, 443-455 (2007). 
38. A. R. Burns, W. Zac Stephens, K. Stagaman, S. Wong, J. F. Rawls, K. Guillemin, B. J. Bohannan, Contribution of neutral processes to the assembly of gut microbial communities in the zebrafish over host development. ISME Journal 10, 655-664 (2016).

39. D. Sprockett, tyRa: Build Models for Microbiome Data. GitHub repository (available at https://danielsprockett.github.io/tyRa/articles/tyRa.html). (2020).

40. J. Oksanen, F. G. Blanchet, M. Friendly, R. Kindt, P. Legendre, D. McGlinn, P. R. Minchin, R. B. O'Hara, G. L. Simpson, P. Solymos, M. Henry, H. Stevens, E. Szoecs, H. Wagner, vegan: Community Ecology Package. R package version 2.5-7. (2020).

41. S. N. Wood, Stable and Efficient Multiple Smoothing Parameter Estimation for Generalized Additive Models. Journal of the American Statistical Association 99, 673686 (2004).

42. S. N. Wood, Fast stable restricted maximum likelihood and marginal likelihood estimation of semiparametric generalized linear models. Journal of the Royal Statistical Society: Series B (Statistical Methodology) 73, 3-36 (2011).

43. S. N. Wood, Generalized Additive Models: An Introduction with R, Second Edition (CRC Press, 2017).

44. A. L. Hicks, K. J. Lee, M. Couto-Rodriguez, J. Patel, R. Sinha, C. Guo, S. H. Olson, A. Seimon, T. A. Seimon, A. U. Ondzie, W. B. Karesh, P. Reed, K. N. Cameron, W. I. Lipkin, B. L. Williams, Gut microbiomes of wild great apes fluctuate seasonally in response to diet. Nat Commun 9, 1786 (2018).

45. J. D. Orkin, F. A. Campos, M. S. Myers, S. E. Cheves Hernandez, A. Guadamuz, A. D. Melin, Seasonality of the gut microbiota of free-ranging white-faced capuchins in a tropical dry forest. ISME J 13, 183-196 (2019).

46. A. Baniel, K. R. Amato, J. C. Beehner, T. J. Bergman, A. Mercer, R. F. Perlman, L. Petrullo, L. Reitsema, S. Sams, A. Lu, N. Snyder-Mackler, Seasonal shifts in the gut microbiome indicate plastic responses to diet in wild geladas. Microbiome 9, 26 (2021).

47. J. P. Mellard, P. Audoye, M. Loreau, Seasonal patterns in species diversity across biomes. Ecology 100, e02627 (2019).

48. A. H. Moeller, S. Foerster, M. L. Wilson, A. E. Pusey, B. H. Hahn, H. Ochman, Social behavior shapes the chimpanzee pan-microbiome. Science Advances 2, e1500997 (2016).

49. O. Kolodny, M. Weinberg, L. Reshef, L. Harten, A. Hefetz, U. Gophna, M. W. Feldman, Y. Yovel, Coordinated change at the colony level in fruit bat fur microbiomes through time. Nature Ecology \& Evolution 3, 116-124 (2019).

50. S. Lax, D. P. Smith, J. Hampton-Marcell, S. M. Owens, K. M. Handley, N. M. Scott, S. M. Gibbons, P. Larsen, B. D. Shogan, S. Weiss, J. L. Metcalf, L. K. Ursell, Y. VazquezBaeza, W. Van Treuren, N. A. Hasan, M. K. Gibson, R. Colwell, G. Dantas, R. Knight, J. A. Gilbert, Longitudinal analysis of microbial interaction between humans and the indoor environment. Science 345, 1048-1052 (2014).

51. A. Gonzalez, J. A. Navas-Molina, T. Kosciolek, D. McDonald, Y. Vazquez-Baeza, G. Ackermann, J. DeReus, S. Janssen, A. D. Swafford, S. B. Orchanian, J. G. Sanders, J. Shorenstein, H. Holste, S. Petrus, A. Robbins-Pianka, C. J. Brislawn, M. Wang, J. R. Rideout, E. Bolyen, M. Dillon, J. G. Caporaso, P. C. Dorrestein, R. Knight, Qiita: rapid, web-enabled microbiome meta-analysis. Nat Methods 15, 796-798 (2018). 